Revue de droit comparé du travail et de la sécurité sociale

3| 2018

La participation des travailleurs dans la grande entreprise privée et publique

\title{
République Démocratique du Congo
}

Patty Kalay Kisala

\section{OpenEdition}

Journals

Édition électronique

URL : https://journals.openedition.org/rdctss/1918

DOI : 10.4000/rdctss. 1918

ISSN : 2262-9815

Éditeur

Centre de droit comparé du travail et de la sécurité sociale

Édition imprimée

Date de publication : 1 novembre 2018

Pagination : 132-135

ISSN : $2117-4350$

\section{Référence électronique}

Patty Kalay Kisala, «République Démocratique du Congo », Revue de droit comparé du travail et de la sécurité sociale [En ligne], 3 | 2018, mis en ligne le 01 novembre 2021, consulté le 13 novembre 2021. URL : http://journals.openedition.org/rdctss/1918; DOI : https://doi.org/10.4000/rdctss.1918

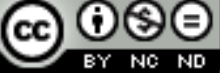

Revue de droit comparé du travail et de la sécurité sociale est mise à disposition selon les termes de la Licence Creative Commons Attribution - Pas d'Utilisation Commerciale - Pas de Modification 4.0 International. 


\title{
PATTY KALAY KISALA
}

\author{
Université Protestante au Congo, Kinshasa
}

\section{I - RÉÉVALUATION DU SMIG}

Le Salaire Minimum Interprofessionnel Garanti (SMIG) en République Démocratique du Congo (RD Congo) ne répondant plus aux conditions socioéconomiques du moment ${ }^{1}$, le Conseil National du Travail (CNT) avait recommandé lors de sa $33^{\text {ème }}$ session tenue à Kinshasa en fin d'année $2017^{2}$ un réajustement raisonnable du taux du SMIG à hauteur de 7075 francs congolais ${ }^{3}$ par jour ${ }^{45}$. Le SMIG congolais a donc été revu à la hausse en mai 2018 par la voie du Décret n 18/017 du 22 mai 2018 portant fixation du salaire minimum interprofessionnel garanti, des allocations familiales minima et de la contrevaleur du logement confirmant le taux de 7075 francs congolais par jour pour un travailleur manœuvre ordinaire ${ }^{6}$.

Le Décret prévoit que le nouveau SMIG s'appliquera graduellement en trois étapes (paliers) avec une augmentation tous les 6 mois à raison de $25 \%$ par palier ${ }^{7}$. Ainsi, le

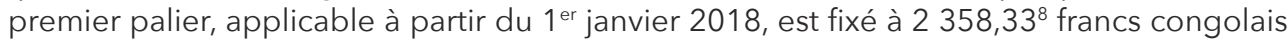
par jour. Le deuxième palier, applicable à partir du 1 er juillet 2018, est fixé à 4 716, 669 francs congolais par jour. Et finalement, au troisième palier, le SMIG pourra être effectivement payé au taux prévu (soit 7075 francs congolais par jour) à partir du 1 er janvier 2019. Pour le secteur agro-industriel, l'évolution du paiement du SMIG se fera à travers quatre paliers ${ }^{10}$, ce qui fait que le taux plein (7075 francs congolais) sera d'application à partir du 1 er juillet 2019 dans ledit secteur ${ }^{11}$.

Le texte précité indique également que le taux du montant journalier des allocations familiales par enfant est arrêté à 1/27 ème par enfant de celui du SMIG du manœuvre ordinaire. De plus, le montant journalier de la quotité saisissable par l'employeur au titre de contre-valeur du logement équivaudra à $1 / 5^{\text {ème }}$ du taux journalier des allocations familiales.

1 Depuis le dernier Smig qui équivalait à près de trois dollars en 2008, les travailleurs avaient perdu au moins $50 \%$ de leur pouvoir d'achat.

http://www.rfi.fr/afrique/20170323-rdc-salaires-smig-hausse-kuku

2 La $33^{\text {ème }}$ session du Conseil National du Travail s'est tenue à Kinshasa du 25 octobre au $1^{\mathrm{er}}$ novembre 2017.

33,70 euros.

4 http://acpcongo.com/acp/smig-passe-de-1-680-a-7-075-fcjour-a-partir-1er-janvier-2018/

5 https://www.digitalcongo.net/article/5ae9a1ea607aa000042182f2/

6 Cf. Art. 2 du Décret n 18/017 du 22 mai 2018.

7 Cf. Art. 3 du Décret n 18/017 du 22 mai 2018.

81,23 euro.

92,47 euros.

101768,75 francs congolais à partir du 1er janvier $2018 ; 3$ 537,50 francs congolais à partir du

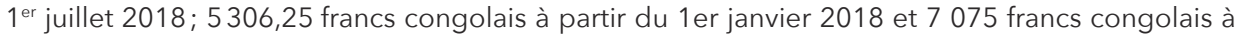
partir du $1^{\text {er juillet } 2019 .}$

11 Cf. Art. 3 du Décret n 18/017 du 22 mai 2018. 
Le Décret prévoit en outre que le taux du salaire soit majoré de $3 \%$ au moins par année entière de service ininterrompu passée par le travailleur dans la même entreprise. La commission tripartite chargée du suivi de l'application du SMIG instituée en $2005^{12}$ devra se réunir au début de chaque année en vue de son éventuel ajustement.

Bien que les syndicats aient contesté la validité d'une note circulaire ${ }^{13}$ du ministre du

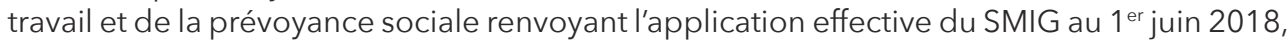
cette initiative a été saluée par la majorité des représentants des travailleurs ${ }^{14}$. II faudrait, toutefois, veiller à une mise à jour régulière du SMIG compte tenu des dépréciations récurrentes du franc congolais et des variations des prix des denrées de première nécessité et afin que le SMIG puisse réellement répondre aux préoccupations sociales des travailleurs en RD Congo.

\section{II - ENTRÉE EN VIGUEUR DU NOUVEAU RÉGIME GÉNÉRAL DE SÉCURITÉ SOCIALE}

Le nouveau régime général de Sécurité sociale prévu par la loi n 16/009 du 15 juillet 2016 est entré en vigueur le 15 juillet 2018 soit vingt-quatre mois après la promulgation du texte comme prévu par l'article 134. La nouvelle loi sur la sécurité sociale entraîne également la création d'un nouvel établissement public à caractère technique et social devant reprendre la gestion du régime général de sécurité sociale ${ }^{15}$. C'est ainsi que la Caisse nationale de Sécurité sociale (CNSS) a été créée en juillet 2018 par Décret du Premier Ministre ${ }^{16}$. Cette institution succède à I'Institut national de Sécurité sociale (INSS) $œ u v r a n t$ depuis $1961^{17}$ à la gestion de la caisse de sécurité sociale.

Le nouveau régime général de Sécurité sociale applicable à compter du 15 juillet 2018 a apporté quelques innovations ${ }^{18}$. On remarquera que la liste des bénéficiaires du régime général s'est élargie puisque le régime est désormais applicable aux mandataires actifs des sociétés d'Etat ou d'économie mixte, aux assurés volontaires, aux employés locaux des missions diplomatiques, aux associés actifs des sociétés, ou aux détenus exécutant un travail périlleux. Les élèves et les étudiants des établissements d'enseignement technique professionnel et artisanal ou les stagiaires en formation sont aussi pris en compte ${ }^{19}$.

12 Arrêté Ministériel n 12/CAB.MIN/TPS/096/05 du 31 août 2005 portant création de la Commission tripartite chargée du suivi de l'application du SMIG.

13 Cf. Note circulaire explicative 003/CAB /MINETAT/MTEPS/FBM/01/2018 du 28 juin 2018 relative à l'application du SMIG fixé par le Décret n 18/017 du 22 mai 2018 du Premier Ministre, Chef du Gouvernement.

14 http://zoom-eco.net/secteur-public/rdc-smig-tshibala-autorise-a-signer-le-decret-fixant-lenouveau-taux-journalier/

15 Art. 8 de la loi n 16/009 du 15 juillet 2016.

16 https://laprosperiteonline.net/2018/07/15/regime-de-securite-sociale-en-rdc-inss-mue-en-cnssagnes-mwad-recolte-le-premier-resultat-de-la-reforme/

17 Cf. Décret-Loi organique de la Sécurité sociale du 29 juin 1961.

18 https://www.inss.cd/2017/05/03/le-dg-de-linss-agnes-mwad-nawej-katang-sensibilise-la-fec-surla-vulgarisation-de-la-nouvelle-loi/

19 Art. 3, 4 et 5 de la loi n 16/009 du 15 juillet 2016. 
Au niveau des prestations, il faut relever la généralisation du droit aux allocations familiales pour toutassuré du régime général pour chacun des enfants à charge ${ }^{20}$. Egalement, au niveau de la branche des prestations aux familles, on mentionnera l'institution du droit aux allocations prénatales pour toute femme assurée ou conjointe d'un travailleur assuré à compter du jour de la déclaration de la grossesse à l'établissement public ${ }^{21}$. De même, une allocation de maternité est prévue à la naissance de l'enfant ${ }^{22}$. Outre les allocations précitées, la femme assurée pourra percevoir une indemnité journalière à l'occasion du congé de maternité. Celle-ci est accordée pendant une période de quatorze semaines dont six avant la date présumée de l'accouchement et huit semaines après l'accouchement à condition que l'assurée cesse toute activité pendant le congé de maternité.

Au niveau de la branche des risques professionnels, on soulignera qu'un accent particulier est mis sur la nécessité de mettre en œuvre des programmes de prévention des accidents du travail et des maladies professionnelles ${ }^{23}$. Le nouveau régime de sécurité sociale prévoit l'allongement du délai de déclaration d'un accident de travail, lequel est porté à 60 jours. Quant au délai de déclaration d'une maladie professionnelle, il court jusqu'à 120 jours. La qualification des maladies professionnelles ne dépendra plus d'une liste limitative et « discriminante » de maladies identifiées dans un tableau. Aussi, d'autres maladies directement causées par le travail peuvent-elles être qualifiées de "maladies professionnelles » après validation d'un " comité de santé » composé d'un médecin de la CNSS, du médecin traitant de la victime et d'un expert désigné par le ministère de la santé ${ }^{24}$.

Au niveau de la branche des pensions, il faut signaler l'uniformisation de l'âge de départ à la retraite à 60 ans pour les hommes et les femmes ${ }^{25}$. L'assuré devra, pour ce faire, avoir accompli 180 mois (15 ans) de cotisation et avoir cessé toute activité salariée. L'assuré atteignant 60 ans sans avoir justifié 180 mois de cotisation à la CNSS bénéficie d'une allocation unique. La mise à la retraite ne peut intervenir qu'à la demande expresse du travailleur. Toutefois, à 65 ans, l'assuré peut être mis d'office à la retraite ${ }^{26}$.

La loi du 15 juillet 2016 prévoit la désignation par la CNSS de contrôleurs habilités à s'assurer du respect des prescriptions du nouveau régime de sécurité sociale par les employeurs et les travailleurs. Ceux-ci jouissent du droit de libre entrée et de libre visite sur les chantiers et dans les locaux des entreprises et peuvent interroger les travailleurs sur l'organisation de l'entreprise par rapport aux normes de sécurité sociale devant être assurées en milieu professionnel.

20 Art. 48,49, et 53 de la loi nº 16/009 du 15 juillet 2016.

21 Art. 39 à 42 de la loi n¹6/009 du 15 juillet 2016.

22 Art. 43 à 45 de la loi n¹6/009 du 15 juillet 2016.

23 Art. 61 de la loi n¹6/009 du 15 juillet 2016.

24 Art. 60 la loi n¹6/009 du 15 juillet 2016.

25 Art. 82 de la loi n¹6/009 du 15 juillet 2016.

26 Art. 84 de la loi n¹6/009 du 15 juillet 2016. 


\section{République Démocratique du Congo}

La CNSS a organisé en mai 2018 des journées portes ouvertes autour de la réforme consacrée par la loi n 16/009 du 15 juillet 2016 fixant les règles relatives au régime général de la sécurité sociale. Cela s'est inscrit dans le cadre de sa campagne de vulgarisation et de sensibilisation de la nouvelle loi de sécurité sociale entrée en vigueur, comme indiqué ci-dessus, le 15 juillet $2018^{27}$.

27 https://www.cnss.cd/2018/05/25/journees-portes-ouvertes-autour-de-la-nouvelle-loi-de-securitesociale/ 